\title{
Thrombectomy aspiration device geometry optimization for removal of blood clots in cerebral vessels
}

\author{
C. Talayero ${ }^{1 *}$, G. Romero ${ }^{1}$, G. Pearce 2 , J. Wong ${ }^{3}$ \\ ${ }^{1}$ Escuela Técnica Superior de Ingenieros Industriales de Madrid, Universidad Politécnica de Madrid (UPM), C/José Gutiérrez Abascal 2 , \\ 28006, Madrid, Spain. Phone: +34913363115 \\ ${ }^{2}$ Department of Mechanical Engineering, School of Engineering, University of Birmingham, Birmingham, B15 2TT, United Kingdom \\ ${ }^{3}$ Department of Cardiac, Thoracic \& Vascular Surgery, National University Heart Centre Singapore, Singapore
}

\begin{abstract}
A study involving the removal of blood clots in cerebral vessels by aspiration thrombectomy is presented. A robust design for the distal end geometry of a catheter is obtained that, together with adequate suction conditions, could avoid potential damage in the artery or fragmentation the thrombus. The optimization process of the parameters is undertaken by a Design of Experiments (DOE) that has been prepared based on Robust Design theories. In particular, 27 experiments are run for one factor at 9 levels (catheter geometry) and up to 9 factors at 3 levels. The experiments are formulated with virtual models that are solved with computing tools. Cosimulation between Computer Fluid Dynamics (CFD) and Finite Elements Method (FEM) structural analysis was used to obtain the suction conditions and the behavior of the blood clot during the intervention process. By comparing the results of the 27 experiments, the highest values of the suctioning force are obtained for a hole pattern based catheter design, that also gives the lowest risk for clot damage (based on the stress value obtained). Direct aspiration and designs based on conical catheter distal ends, give less robust solutions (results are not stable when the conditions of the environment change). Our study investigated the distance between the catheter and the clot, and it was noted that if the catheter was far from the clot, the suction generated a vessel narrowing and consequent potential damage. Up to $90 \mathrm{kPa}$ could be applied when suctioning at a maximum distance equal to the diameter of the vessel between the distal end of the catheter and the proximal end of the clot. A maximum suctioning force of $0,514 \mathrm{~N}$ was achieved without damage to the artery or the clot. This research enables us to determine and use the most representative parameters and geometries to be tested in in-vitro and in-vivo experiments. In this virtual study, hypothesizes are assumed with regard to the material properties, but the robustness of the design process allows to expect similar results in future in-vitro and in-vivo tests.
\end{abstract}

ARTICLE HISTORY

Revised: $18^{\text {th }}$ Nov 2019

Accepted: $18^{\text {th }}$ Nov 2019

\section{KEYWORDS}

Blood clot removal; aspiration Thrombectomy device;

robust design;

stroke

\section{INTRODUCTION}

Stroke is one of the commonest causes of death globally [1]. Ischemic strokes are more common than hemorrhagic strokes and these arise in the brain when the blood supply to an area of the brain becomes interrupted or cut off e.g. when a blood clot occludes a vessel in the brain. The arteries that comprise the Circle of Willis in the human brain are among the vessels that are most frequently affected by stroke. In recent years, several devices and techniques have been employed in blood clot removal [2-4]. Mechanical Thrombectomy Devices (MTD) [5-7] have been compared to Aspiration Thrombectomy Devices [8-10] and the effectiveness of using both in combination has also been investigated [11]. Some studies [2] have indicated that modern aspiration devices give better results concerning the recanalization of cerebral vessels in patients suffering from acute ischemic stroke when compared to first generation MTDs and third generation stent retrievers, especially in the case of small size vessels such as those found in the Circle of Willis (cerebral vessels). Techniques are currently under development which are concerned with the optimization of different suctioning mechanisms (alternative pumps, continuous suctioning or manual suction with syringes) and which also involve several distal end shapes and components [12-14]. An extraction device named the GPTAD (GP Thrombus Aspiration Device) $[15,16]$ was designed in an attempt to overcome some of the potential problems involved in the medical intervention. The device may potentially reduce the risk of downstream embolization since it does not have to make contact with the clot, in order to enable blood clot extraction and it has no moving parts and thus potentially the risk of breakages occurring in the device. In-vitro studies undertaken by Tennucci et al [17] have also shown that it potentially reduces the risk of clot fragmentation. A schematic view of an aspiration thrombectomy is shown in Figure 1. 


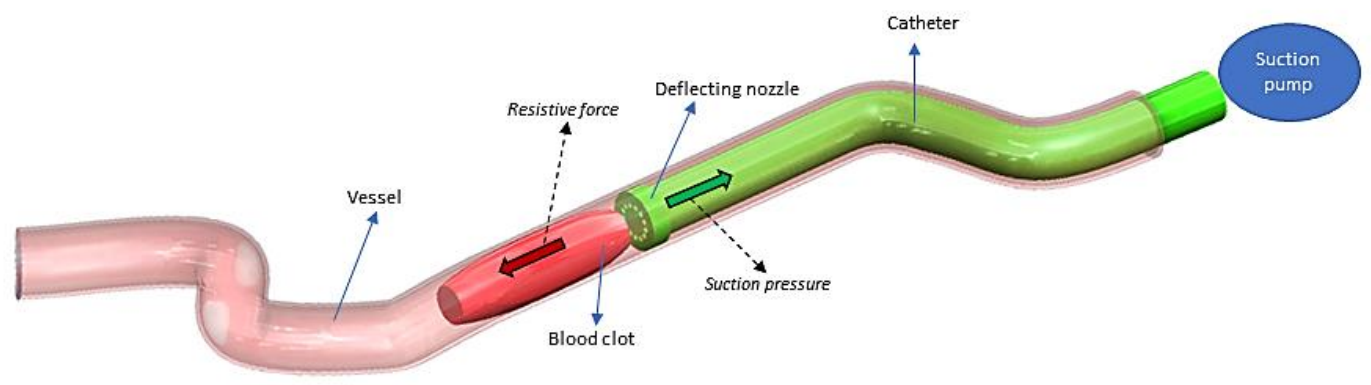

Figure 1. Schematic view of the GPTAD behavior.

Optimization of the process of clot removal and geometrical design of the clot extraction device is of great importance. The study we present aims to research the behavior of the thrombus by using virtual models, and to investigate the minimization of the stress that can give rise to clot fragmentation, which then can potentially increase the risk of downstream embolism. In the medical intervention, called thrombectomy, some parameters can be set and affect the efficiency of clot extraction, such as the suction pressure, de size of the catheter, the nozzle (distal end of the catheter) or the distance between the catheter and the blood clot. On the other hand, other parameters related to the patient can also affect efficiency of the thrombectomy: size of the thrombus (length and diameter), the contact area between the thrombus and the artery, or the mechanical properties of the thrombus and the artery, among others [18]. We therefore undertake a Design of Experiments (DOE) to ensure that the correct number of experiments are involved in order to identify the influence of these various factors. Based on the properties of orthogonal matrices, the concepts of Robust Design are applied to reduce the signal to noise ratio or, in other words, to obtain a design that gives good results (signal) independently of the non-controllable factors (noise). The diameter of the artery used in the virtual testing is typically 3 mm diameter (Circle of Willis vessels size).

\section{METHODS}

\section{Design of the Experiment}

Typical DOE are based on factorial analysis where $\mathrm{k}$ factors at two levels are considered. In the case studied and because of the wide range of some factors, more levels are required. For these studies, predefined $L$ orthogonal matrix are useful since a high number of levels can be studied with a reduced number of experiments. An optimization in two stages was undertaken. In the first step, the variability was minimized by combining up to 10 parameters without interaction factors. The aim of this was to obtain the best geometry for the distal end of the catheter (i.e. which was most stable against external factors and produced good mechanical behavior). In the second stage, the resultant geometry was adjusted to obtain as good a result as possible (mean adjustment).

A total of 27 tests were completed by using a $L_{27}($ b) matrix, where one factor is studied at 9 levels, plus nine factors at 3 levels. This combination of parameters guaranteed the minimum independent number of tests needed. The combination of this number of factors and levels used in another way would give redundant experiments or interactions instead of factors, needing $9 \times 3^{9}$ experiments in order to reach all the possible combinations. Since the chosen matrix does not have columns dedicated for interactions, all the studied factors could be added to the matrix.

The chosen factor at nine levels is the catheter design itself, since it is the one that offers a greater variety of different options to be designed. The designs have been undertaken with due consideration being given to the manufacturability and the absence of rigid parts that could be hazardous during the thrombectomy. We began with a simple design where the aspiration is directly done by a cylindrical catheter, in accordance with the designs shown in the patents US2015133973A1, US2013060206A1, US2015005792A1 and US2009171368A1. We also considered other designs which formed the basis for the geometries studied, namely, conical based catheters, a helix based catheter and a hole patterned catheter were designed as represented in Figure 2. 


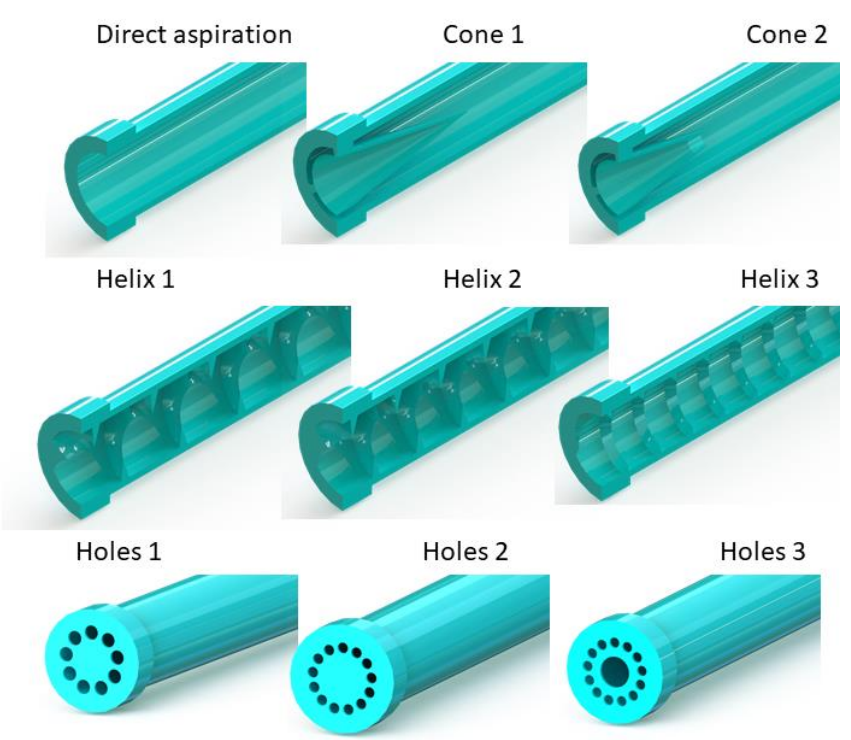

Figure 2. Catheter 3D models used in the virtual tests.

The parameters that define each design are: the slope of the cone and the free area of suction for the tapered solution; the pitch, the depth and the shape of the helix for the helical catheter; and the number, diameter and distribution of the holes for the whole patterned solution. The remaining factors of the experiments were established at three levels as in Table 1.

Table 1. DOE factors and values.

\begin{tabular}{lc}
\hline Factor & Value of the levels \\
\hline Suction pressure (kPa) & $20-30-40$ \\
$\begin{array}{l}\text { Distance between de distal end of the catheter and } \\
\text { the clot (mm) }\end{array}$ & $0-2-4$ \\
Difference in transversal section between the & $0-5-10$ \\
vessel and the catheter (\%) & $3-5-10$ \\
Clot length (mm) & $0.05-0.1-0.15$ \\
Clot Young Modulus (MPa) & $0.4-0.45-0.499$ \\
Clot Poisson coefficient & $0.5-0.8-1.1$ \\
Vessel Young Modulus (MPa) & $80-90-100$ \\
Vessel occlusion (\%) & $0.01-0.05-0.1$ \\
Occlusion force (N) &
\end{tabular}

Some of the factors could be adjusted in the actual intervention involving clot removal itself, such as the suction pressure and the distance between the distal end of the catheter and clot. However, other factors are related to the clot properties, such as the length, the Young Modulus and the Poisson coefficient. The important factor involving the vessel is its Young Modulus. Finally, two factors that relate the vessel and the clot are considered: the area of the vessel occluded by the clot and the occlusion force, i.e. the force that needs to be achieved or superseded by the suction process in order to cause clot movement.

\section{Parameters Definition}

In preliminary studies [19] the clot removal was modelled in Bond Graph paying attention to the whole system (artery - clot and the interfaces). In those studies, the suction pressure vs time was the result that was analyzed, and the clot itself was not analyzed from the point of view of its stress. Using these results (the suction pressure values) and bearing in mind the need to analyze any possible damage to the blood clot, a model to represent the internal behavior of the clot was considered. In this model, the clot is modelled as a several mass - spring system that represents "slices" of the clot that can be studied with respect to strain and stress, where the force over the mass is variable in time, $\mathrm{F}(\mathrm{t})$ along the direction $\mathrm{x}(\mathrm{t})$. This direction is the only direction under consideration regarding clot movement. In this way we obtained a Multiple Degree of Freedom system (MDOF). 


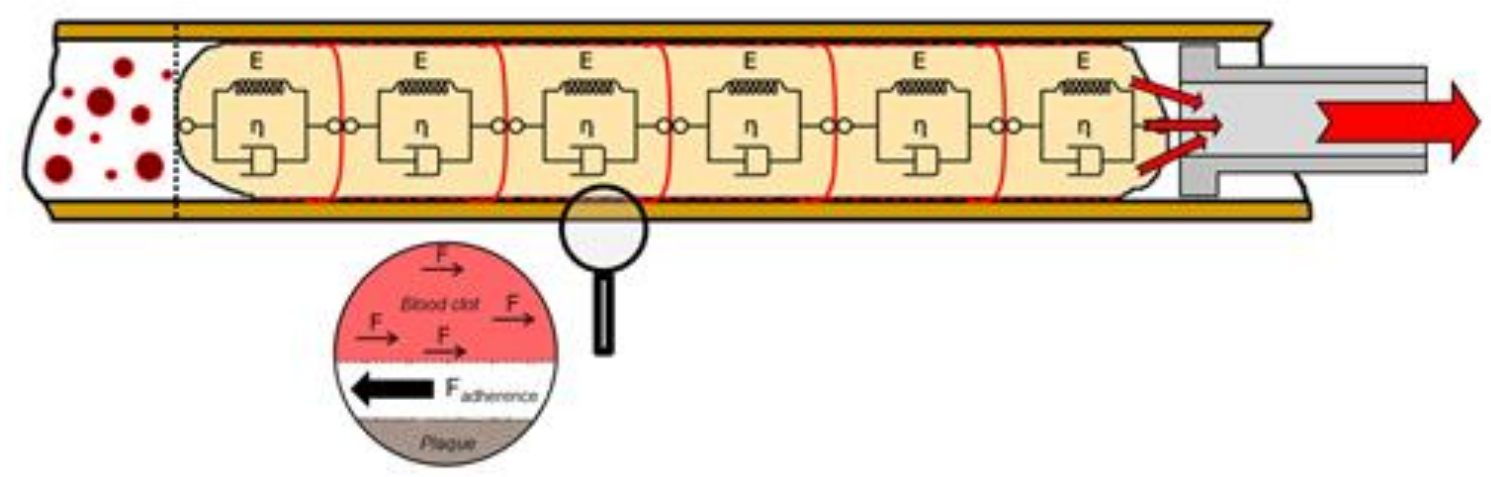

Figure 3. Damped mass-spring system with 6 DOF.

Solving this model allowed adjustment (in a very rapid manner) of the clot properties (Young's Modulus, Poisson coefficient and Yield limit) based on the experimental in-vitro testing. In this model, the input variable is the suction pressure that can vary in a given period of time until a steady value is achieved. We used typical clinical data in the study, e.g. suction pressure and clot size, that have been used in previous Bond Graph studies [20]. In around 60 seconds, a mean value of $50 \mathrm{kPa}$ was reached, considering that, the longer the clot, the higher the needed time (for a $30 \mathrm{~mm}$ clot, the pressure required for clot extraction was reached in 50 seconds, while a $50 \mathrm{~mm}$ clot needed almost 90 seconds).

The suction itself was represented by a combination of values of axial load and torsional load, assuming that it is possible to create such a torque with a catheter with a helix geometry such as the GPTAD in in-vitro tests. In addition, the Young Modulus was calculated from the stiffness of the thrombus, a variable value depending on the elongation, and ranged between 0.1 and $0.5 \mathrm{~N} / \mathrm{mm}$ based on experimental testing on porcine thrombus.

\section{Experiments Run}

Once we had defined the range of the mechanical properties, and in order to run the 27 defined experiments, a threedimensional model was required in order to accurately represent the geometry of the catheter, that is the most significant limitation of the Bond Graph or Simulink models. For that, nine catheter geometries were created and assembled in the virtual models.

To solve the experiments, the medical intervention methodology was reproduced in a 3D assembly created with SolidWorks software, with a cylindrical tube that represented the artery, a non-regular revolution solid to represent the blood clot, and the catheter geometry.

The sequence for doing the experiments was as follows: prepare the initial geometry, solve the fluids problem (suctioning with a given pressure), and solve the mechanical problem (with the results of the fluids study, the stress, strain and displacements were calculated).

The co-simulation algorithm needs to automatically use the data from the CFD to be used in the mechanical problem as an input (the pressure field and shear loads). Since the geometry is changing from the initial condition for each instant of time (which impacts on the results calculated for the suction), an updated geometry is created automatically for each time step [21], where the clot displacement and deformation are the resultant of the previous time step.

In addition, the clot is a mass that has usually formed in another location e.g. in the heart or another vascular artery (typically the carotid artery) and has become dislodged becoming trapped in smaller diameter artery, such as the cerebral artery (considered in this work). For that reason, the blood clot will not be considered as a fluid (blood is usually studied as a Non-Newtonian fluid) but an hyperelastic solid. The hyperelastic material models can be used to model materials such as rubber, where the solutions involve big strains. The clot is therefore considered to be an elastic material, nonlinear, isotropic and incompressible.

For the mesh of the thrombus and the vessel, tetrahedral elements (TETRA10) were used with a size that adequately represented the geometry and that correctly reproduced the bending effects, especially in the artery. The chosen high order elements also provided better stability in the hyperelastic model than lower order elements (TETRA 4). Additionally, we noted that a coarse mesh generated a stiffer system that did not represent adequately the aspiration thrombectomy intervention.

SolidWorks Flow Simulation and SolidWorks Nonlinear Dynamics were chosen since they allowed us to automate the thrombectomy event during a period of time and to easily modify the geometry. The boundary conditions for the study were:

- Fixed artery on the endings, far enough from the area being studied (artery ending distance to the thrombus is three times the length of the thrombus), to reproduce the strain properly

- Zero initial velocity on the distal thrombus surface

- In addition, an automatic time lapse was chosen, initially using $0.01 \mathrm{sec}$, and varying from 1e-6 to 0.01 seconds; allowing up to 5 iterations on each time step. These dynamic parameters allowed us to simulate the thrombectomy event as it would appear under real conditions. 


\section{RESULT AND DISSCUSSION}

The CFD and FEM analysis produced results based on 3D geometries as shown in Figure 4, where a pressure plot is shown for some catheter designs. The output to control - in our case a continuous variable - was the highest value of the suction force created by the pressure generated by the pump. The suction force indicated if the clot started to move. It was also deemed necessary to control - also a continuous variable) the maximum value of the Von Mises stress in the clot and in the artery, as critical parameters to avoid undesirable damage. The results showed that neither the clot nor the vessel suffered damage with the suction pressures used for any of the suction pressures considered. However, clot movement was not achieved in every experiment. In the case of the direct aspiration catheter, both conical designs and in the case of helix model 2, the force needed to suction the clot was not always reached, due to the pressure losses generated by the geometry which lead to a low suctioning force for the clot.
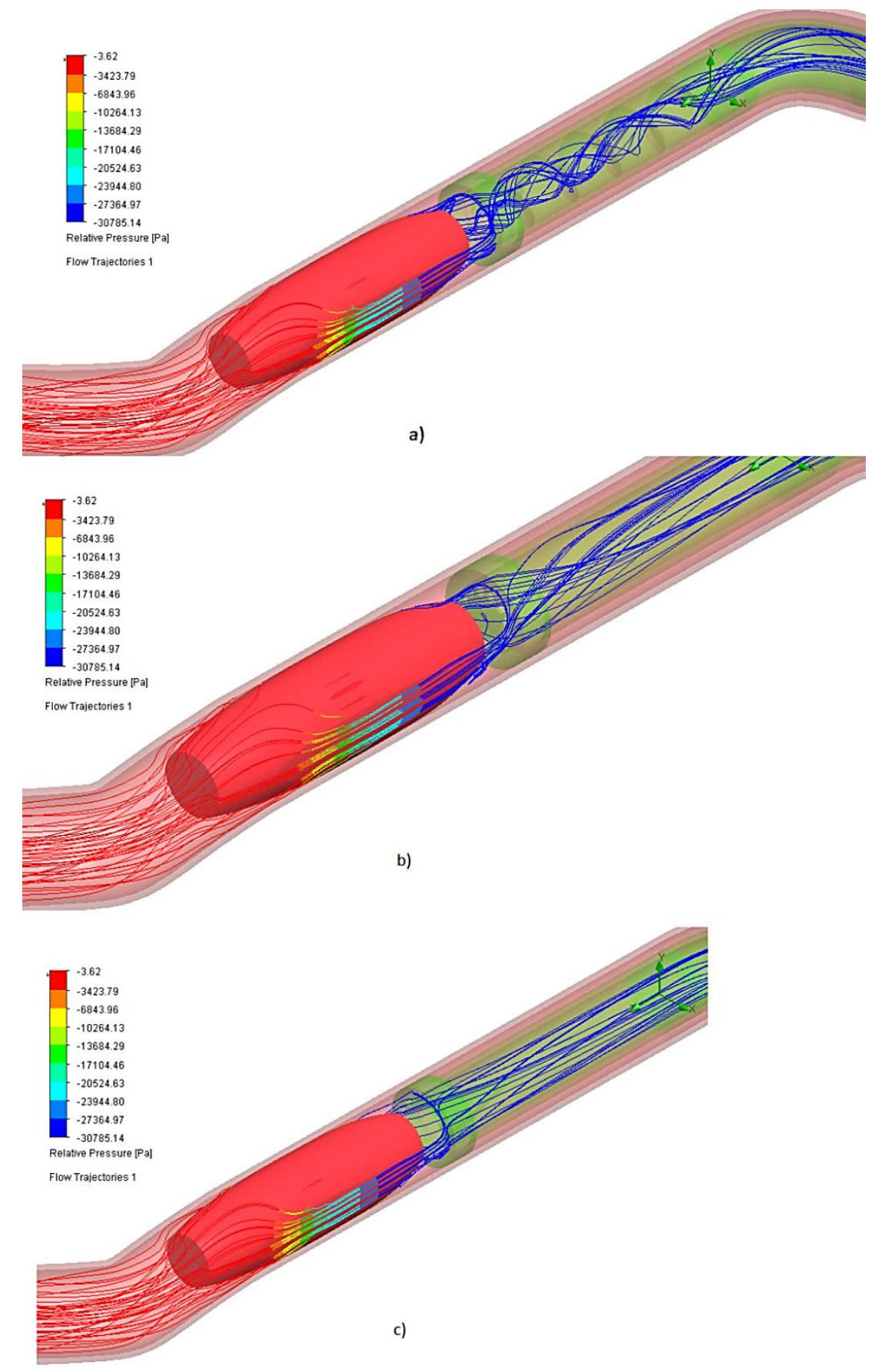

Figure 4. Pressure distribution for a given experiment: (a) Helix, (b) Holes pattern and (c) Cone.

The results were studied by an ANOVA analysis. The influence of the factors that can be set in the medical intervention (they can be optimized) were studied, and the clot geometrical properties (noise to consider). The Multi Factor ANOVA analysis is shown in the Table II taking the suction force as the measured variable. For the chosen 
confidence value (95\%), two parameters are statistically significant to the result: the suction pressure and the clot-vessel occlusion. The second one is a noise variable (we cannot control this), but the suction pressure is a factor that can be totally controlled during the medical intervention.

Table 2. MANOVA for the suction force.

\begin{tabular}{lccc}
\hline \multicolumn{1}{c}{ Term } & Degrees of Freedom & F-Ratio & p-value \\
\hline A: Catheter & 8 & 2.31 & 0.162245 \\
B: Suction pressure & 2 & 14.17 & $0.005333^{*}$ \\
C: Distance & 2 & 3.09 & 0.119258 \\
D: Clot length & 2 & 1.52 & 0.291503 \\
E: Resistive force & 2 & 1.48 & 0.300784 \\
F: Catheter - vessel free area & 2 & 1.93 & 0.225344 \\
G: Clot - vessel occlusion & 2 & 17.89 & $0.002963^{*}$ \\
Total (Adjusted) & 26 & & \\
Total & 27 & & \\
* Term significant at alpha $=\mathbf{0 . 0 5}$ & & & \\
\hline
\end{tabular}

In Figure 5 we show the suction forces for each catheter design (the geometry is shown in Figure 2). Comparing the values by categories, we show that with all of the holes designs we achieve suction forces up to a $100 \%$ higher than with direct aspiration. The percentage is around $70 \%$ when comparing the holes and the cone designs. The differences between the designs are accounted for the velocity and pressure fields that are obtained from the suction pressure. In the case of the cone designs and Direct aspiration, the maximum pressure values are reached in the centerline (revolution axis) of the catheter, achieving very low pressure values at the clot-vessel interface, resulting in a global low suction force.

In the case of the helix designs, the Helix 2 gives lower values due to turbulence generated by an improper pitch and height of the helix. In Helix 1 and 3, the height is in accordance to the pitch, and, instead of turbulence, a twist effect appears (vortex), achieving high values for suction forces.

Finally, the holes designs achieve the best results due to the load concentration at the clot-vessel interface. By applying the load on that area, the effect of the suction is much more effective than if it is applied on the centerline.

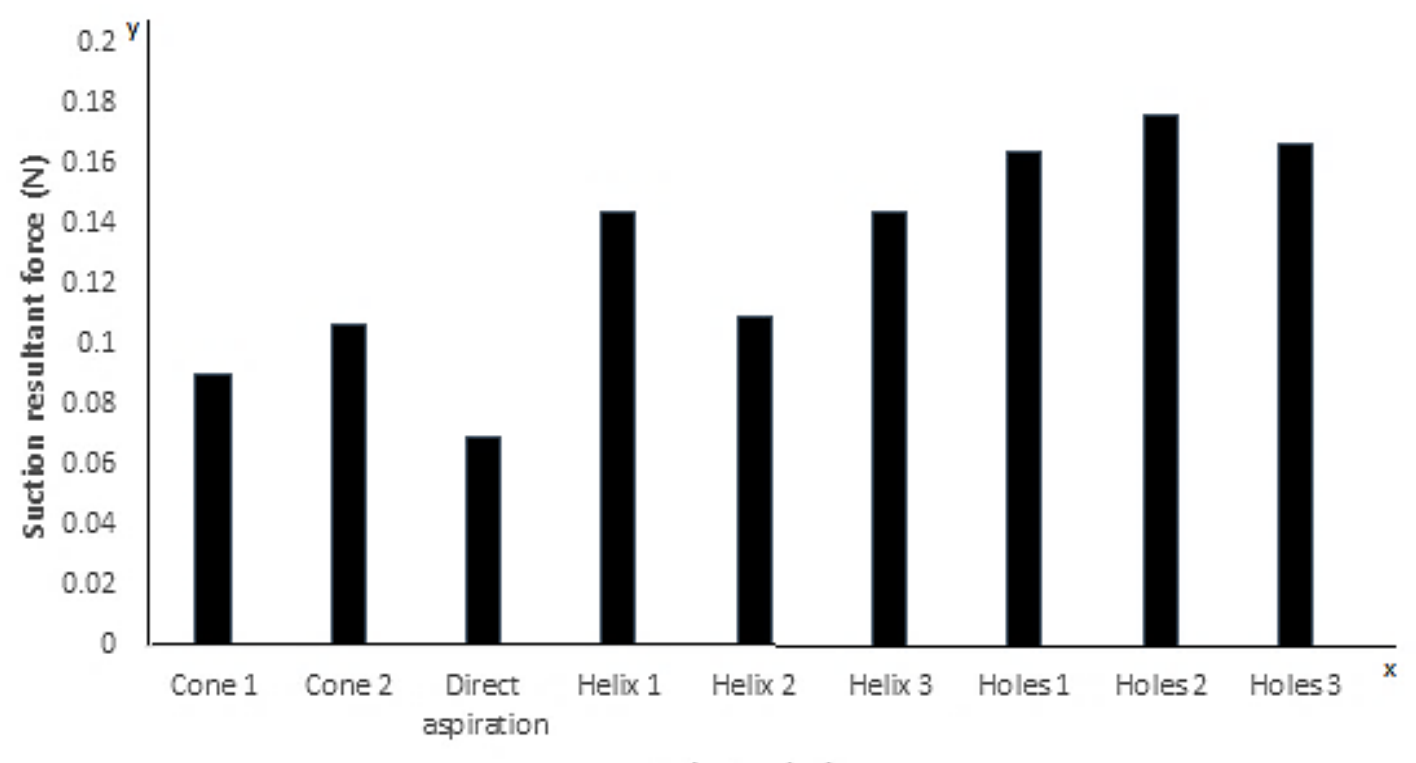

Catheter design

Figure 5. Means plot for catheter geometries.

In Figure 6, the suction force is plotted vs the suction pressure for each group of the tested designs, and for the three levels of the factor $(20-30-50 \mathrm{kPa})$. The highest values of the suctioning force are always obtained for the Hole based designs, independent of the suction pressure. In addition, we note that both the helix-based designs and hole-based designs, give high positive slopes. This behavior is good since it means that, the higher the pressure, the higher the force. In the cone designs, the slope is almost zero, what means that higher pressures will not achieve movement of the clot and could potentially damage the vessel. Finally, we note that for the Direct aspiration solution, a high value of suction pressure could under some circumstances give rise to lower suction forces, which could carry a risk of potential damage 
to the vessel without suction of the blood clot occurring.

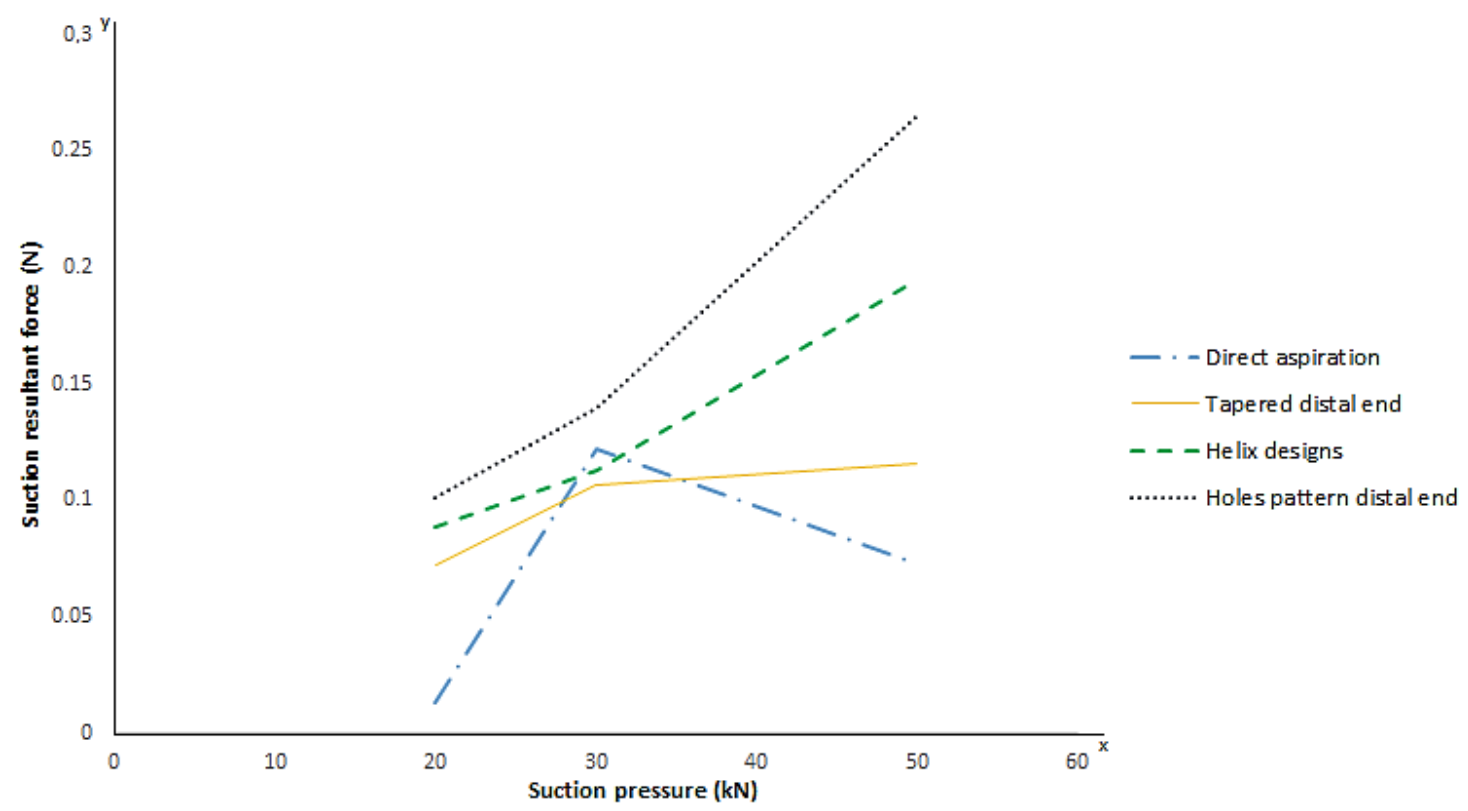

Figure 6. Plot for suction pressure vs force for the series of designs.

Other parameter that can be set up in the intervention is the distance between the catheter and the clot $(0-2$ and 4 $\mathrm{mm}$ for the experiments). We note that if the catheter is far from the clot, the suction generates a vessel narrowing. Consequently, the distance should be closer than the equivalent to the diameter of the vessel for the highest suction values to be applied (Figure 7). In this case, the helix designs give slightly higher values than the hole design, but only in the situation where the distal end of the catheter is in contact with the thrombus (this situation should be avoided to reduce the risk of fragmenting clot). As in the case of suction pressure analysis, the cone designs have an almost flat behavior. It is noteworthy that the suction force is similar for all the designs when the catheter is relatively far from the clot (4 mm). This indicates that the effect caused by the nozzle is lost over large distances (the flow reaches a velocity distribution as in a pipe).

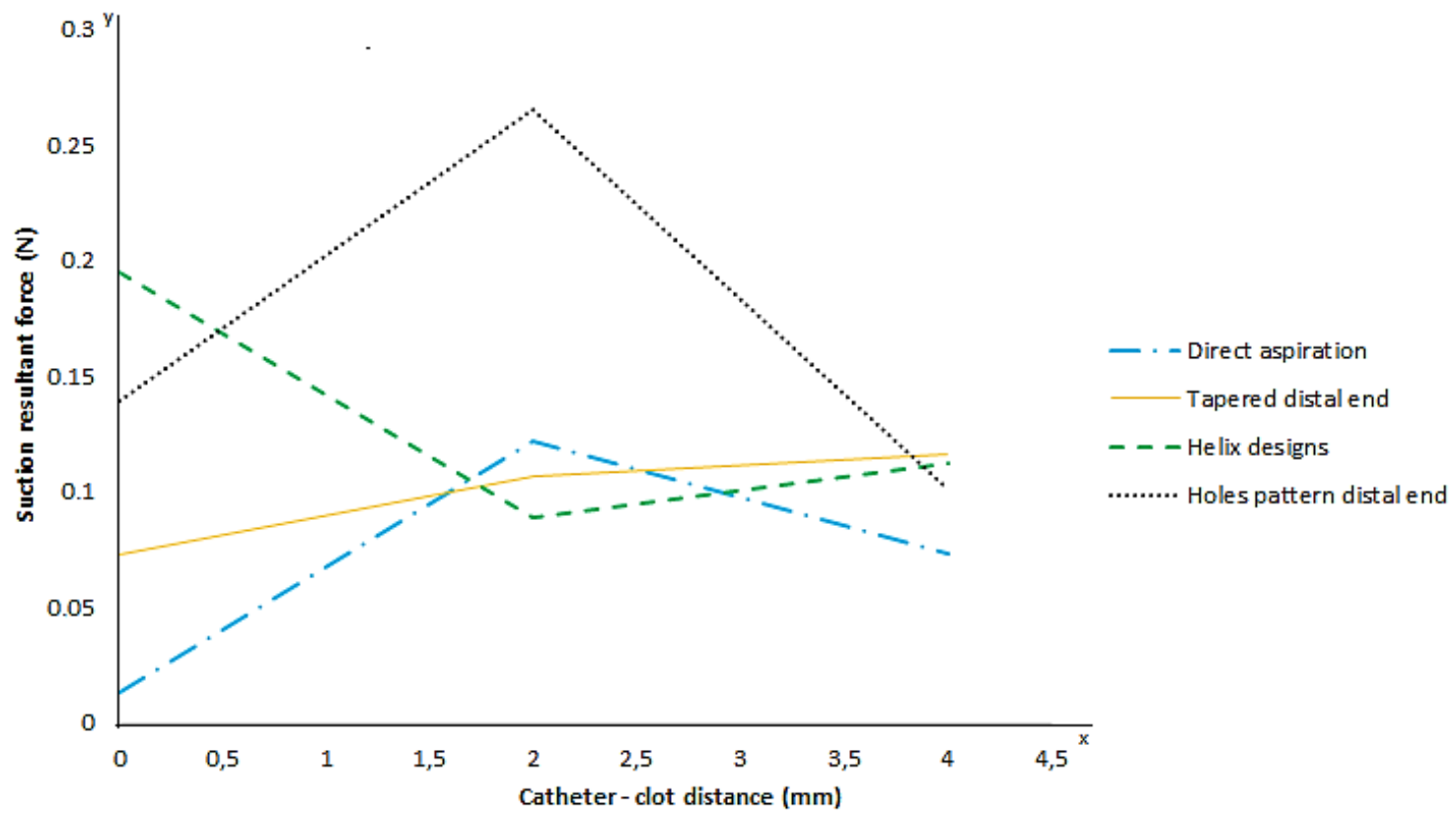

Figure 7. Plot for suction pressure vs clot distance for the series of designs.

Taking the solution that gives the maximum suctioning force (Holes 2 design), the second step optimization was performed by taking an analysis with a suctioning pressure up to $90 \mathrm{kPa}$ (the maximum tested in in-vitro testing) and varying the holes pattern with a $L_{4}$ matrix (three factors at two levels: number of holes, diameter of holes, distance of the holes to the symmetry axis). The results show a maximum suctioning force of $0,514 \mathrm{~N}$ without damage to the artery or the clot. The design has also been virtually tested on a $1 \mathrm{~mm}$ diameter vessel (all the results shown in this study are 
referred to a $3 \mathrm{~mm}$ diameter vessel) giving proportional results and showing the same qualitative behavior.

The results obtained are consistent with previous Bond Graph models and in-vitro experiments, that indicated that the pressure needed to extract a blood clot from an artery are within the range used ( 20-90 kPa). This indicates that aspiration thrombectomy is actually a good option to use in the removal of blood clots in human arteries because it will not potentially cause damage due to the suction operating pressures used. Simple spring-mass models are useful to study the system and provide a good approach to studying the clot behavior (possible damage) during the suction stage (elongation and stress), and the results also permit fast estimation of parameters for more complex studies.

Based on these parameters, complex geometries can be designed and virtually tested with the combination of Computer Fluid Dynamic and Nonlinear Dynamic FEM models to test a multitude of different designs with no risk to patients. In addition, the modelling involves very low resources in terms of time compared to laboratory testing. Once the model is created, the time to obtain a solution is around 60 minutes in a Core i7 processor, so in 27 hours in our case (a little bit more than one day) we could have results that, otherwise, we would need this time to obtain only the nozzles manufactured using 3D printing ( 2 hours are needed in a Fused Filament standard printer). The 3D based models also permit a detailed analysis of suction forces (based on a pressure field) to be undertaken. The results of mathematical models and FEM models are consistent (stresses and elongation). It is significant that the mean solving time for the computer models are around $60 \mathrm{~min}$ in a Core $\mathrm{i} 7$ processor while the spreadsheet is updated in less than a second for new values.

The results concerning the 3D analysis show that the catheter geometry notably affects the pressure losses during aspiration. In some cases (Helix 2, Direct aspiration) the velocity field in the catheter and the nozzle leads to effects that are the opposite than those desired (lower suction force applied on the clot for higher suction pressures). This undesirable effect could potentially cause damage in the vessel due to the high pressure applied.

The pressure distribution in the domain studied (vessel - blood clot - nozzle) also has a bearing on the movement of the clot and the tensional state. The designs that concentrated the highest pressures close to the walls of the artery, achieved higher suction resultant forces. The forces were also applied in a more effective way because they led to lower stress values in the clot when the movement began.

The use of factorial analysis based on orthogonal matrix and robust design (Taguchi method) permits us to combine several parameters in a reduced number of experiments that are not redundant. The techniques involved also allow us to guarantee the robustness of the chosen design, to identify the desired output variable, the control parameters and the noise factors, with the aim of obtaining a design that gives results that are as independent as possible of the uncontrolled factors.

In the cases studied, the most significant factor is one that cannot be set up in the actual interventional process of clot removal (namely, how strongly is the clot occluded in the vessel). Nevertheless, our approach gives an indication of the potential operating parameters that should be used in clot removal based on the clinical data available and the results of previous in-vitro studies. The highest suctioning pressures are preferred for all the possibilities considered with the exception of Direct aspiration; and the highest suction force for the pressure used in that case are those obtained for a distal end with a hole pattern. This is consistent with the mathematical models that indicated that the suctioning force has better effects when applied to the periphery of the blood clot (clot - vessel interface) instead of in the center of the clot or uniformly.

It was also found that the stiffness (Young modulus and Poisson coefficient) and the clot size (noise factors) are not critical when adequate pressure was applied. On the other hand, the effectiveness of the aspiration thrombectomy falls rapidly when the distal end of the catheter is further from the clot more than a distance equivalent to one diameter ( $3 \mathrm{~mm}$ in the case of our experiments). In reality, however, in actual clinical clot extraction from an artery, imaging techniques can be used to determine the distances involved from the end of the catheter to the blood clot itself (medical interventional angiography).

In reality, the size of the catheter used can be matched to the dimensions of the artery containing the blood clot. However, this appears to be of less importance than other factors in terms of producing clot movement and, although some turbulence appears between them, the pressure drop is negligible. The results of our research also comply with manufacturability requirements, keeping high flexibility in the catheter design with no rigid parts that could cause damage in the clot removal intervention.

\section{CONCLUSIONS}

The present study permits concluding that:

- The mechanical values (stress) resulting from the suction pressure required to remove a blood clot in an artery as derived from the mass-spring model are consistent with those produced by experimental investigations and previous studies,

- 3D based models are appropriate for solving these problems where fluids and solids in motion are combined. Cosimulation algorithms allow us to combine the fluid and the mechanical problems.

- Following virtual tests, it is found that the suction pressure needed in aspiration thrombectomy for the clot movement, is small enough to avoid potential damage in the vessels. Up to $90 \mathrm{kPa}$ can be applied when suctioning at a maximum distance equal to the diameter of the vessel between the distal end of the catheter and the proximal end of the clot. The smaller the distance, the better the result.

- For an Aspiration Thrombectomy it is better to use a catheter geometry that generates the suctioning force due to the pressure at the clot - vessel interface. 
- As the virtual tests are repeated over a range of conditions, the qualitative results of physical testing should be similar. The best design obtained in the study should give the best results in physical testing, regardless of the properties of the clot or vessel.

- This type of modelling is useful both in evaluating the performance of existing catheters but also in the formulation, development and testing of new and innovative catheter designs.

\section{REFERENCES}

[1] Scarborough P et al. Stroke statistics. British Heart Foundation and Stroke Association. Department of Public Health, University of Oxford. 2009:12-13.

[2] Spiotta AM, Chaudry MI, Hui FK, Turner RD, Kellogg RT, Turk AS. Evolution of thrombectomy approaches and devices for acute stroke: A technical review. Journal of Neurointerventional Surgery. 2015;7(1):2-7.

[3] Davis S, Lees K, Donnan G. Treating the acute stroke patient as an emergency: current practices and future opportunities. International Journal of Clinical Practicews. 2006;60(4):399-407.

[4] Hesse AC, Behme D, Kemmling A, Zapf A, Hokamp NG, Frischmuth I et al. Comparing different thrombectomy techniques in five large-volume centers: A 'real world' observational study. Journal of NeuroInterventional Surgery 2018; 10(6):525-529.

[5] Gobin YP et al. MERCI 1: A phase 1 study of mechanical embolus removal in cerebral ischemia. Stroke. 2004; 35(12):28482854.

[6] Mokin M, Nagesh SVS, Ionita CN, Mocco J, Siddiqui AH. Stent retriever thrombectomy with the Cover accessory device versus proximal protection with a balloon guide catheter: In vitro stroke model comparison. Journal of Neurointerventional Surgery. 2016; 8(4):413-417.

[7] Bose A et al. The penumbra system: A mechanical device for the treatment of acute stroke due to thromboembolism. American Journal of Neuroradiology. 2008; 29(7):1409-1413.

[8] Son S et al. Comparison of Solitaire thrombectomy and Penumbra suction thrombectomy in patients with acute ischemic stroke caused by basilar artery occlusion. Journal of Neurointerventional Surgery. 2014; 8(1):13-18.

[9] Fanous AA, Siddiqui AH. Mechanical thrombectomy: Stent retrievers vs. aspiration catheters. Cor et Vasa. 2016;58(2):193203.

[10] Lapergue B et al. A direct aspiration, first pass technique (ADAPT) versus stent retrievers for acute stroke therapy: An observational comparative study. American Journal of Neuroradiology 2016; 37(10):1860-1865.

[11] Mokin M, Ionita CN, Nagesh SVS, Rudin S, Levy EI, Siddiqui AH. Primary stentriever versus combined stentriever plus aspiration thrombectomy approaches: In vitro stroke model comparison. Journal of Neurointerventional Surgery. 2015;7(6):453-457.

[12] Bonnette M, Morris D. Thrombectomy catheter system (patent US2014303658A1).

[13] Cosigny P. Spiral thrombectomy catheter (patent US2013060206A1).

[14] Milner K, Barnett A. Thrombectomy catheter with flow directing mechanism (patent US2015133973A1).

[15] Pearce G, Perkinson ND. Biomechanical probe international patent corporate treatise (WO2006120464); European patent (ep1893195 (a2)); Japanese patent (jp2008639924 (t)); Chinese patent (cn101208049 (a)).

[16] Pearce G, Brookfield P, Burley M, Murcott D, Perkinson ND, Spence J, Wong J. The 'GP' mechanical thrombectomy device: Measurements and modelling, using laboratory models, applications of geometric algebraic techniques and cadaveric brains. Journal of Stroke and Cerebrovascular Disease. 2009; 18(4) 288-293.

[17] Tennuci C, Pearce G, Wong J, Nayak S, Jones T, Roffe C. Comparison of the effectiveness of three methods of recanalization in a model of the middle cerebral artery: Thrombus aspiration via a $4 \mathrm{~F}$ catheter, thrombus aspiration via the GP thromboaspiration device and mechanical thrombectomy using the solitaire thrombectomy device. Stroke Research and Treatment. 2011;186424.

[18] Goswami P, Mandal DK, Manna NK, Chakrabarti S. Numerical investigations of various aspects of plaque deposition through constricted artery. Journal of Mechanical Engineering and Sciences. 2019;13(3):5306-5322.

[19] Romero G, Higuera I, Félez J, Pearce G, Perkinson ND. Modelling and simulation of a thrombectomy probe applied to the middle cerebral artery by using the bond graph technique. $9^{\text {th }}$ International Conference on Bond Graph Modeling and Simulation. 2010; 172-179.

[20] Romero G, Martinez ML, Pearce G, Mera JM. An investigation into the performance of a new mechanical thrombectomy device using bond graph modeling: application to the extraction of blood clots in the middle cerebral artery. Simulation. 2013; 89(3):381-391.

[21] Satrio D, Utama IKAP, Mukhtasor. The influence of time step setting on the CFD simulation result of vertical axis tidal current turbine. Journal of Mechanical Engineering and Sciences. 2018;12(1):3399-3409. 